\title{
Erratum: Forecasting societies' adaptive capacities through a demographic metabolism model
}

Wolfgang Lutz and Raya Muttarak

Nature Climate Change 7, 177-184 (2017); published online 2 March 2017; corrected after print 2 March 2017.

Owing to technical problems, this Perspective was published online later than the date given in the print version. The published date should read '2 March 2017', and is correct in the online versions. 